\title{
ANNULUS CRITERIA FOR OSCILLATION OF SECOND ORDER DAMPED ELLIPTIC EQUATIONS
}

\author{
ZHITING XU
}

ABStract. Some annulus oscillation criteria are established for the second order damped elliptic differential equation

$$
\sum_{i, j=1}^{N} D_{i}\left[a_{i j}(x) D_{j} y\right]+\sum_{i=1}^{N} b_{i}(x) D_{i} y+C(x, y)=0
$$

under quite general assumption that they are based on the information only on a sequence of annuluses of $\Omega\left(r_{0}\right)$ rather than on the whole exterior domain $\Omega\left(r_{0}\right)$. Our results are extensions of those due to Kong for ordinary differential equations. In particular, the results obtained here can be applied to the extreme case such as $\int_{\Omega\left(r_{0}\right)} c(x) d x=-\infty$.

\section{Introduction}

Consider the second order damped elliptic differential equation

$$
\sum_{i, j=1}^{N} D_{i}\left[a_{i j}(x) D_{j} y\right]+\sum_{i=1}^{N} b_{i}(x) D_{i} y+C(x, y)=0
$$

in $\Omega\left(r_{0}\right)$, where $x=\left(x_{1}, \ldots, x_{N}\right) \in \mathbb{R}^{N}, D_{i}=\partial / \partial x_{i}$ for all $i, \Omega\left(r_{0}\right)=\{x \in$ $\left.\mathbb{R}^{N}:|x| \geq r_{0}\right\}$ for some $r_{0}>0$, and $|x|=\left[\sum_{i=1}^{N} x_{i}^{2}\right]^{1 / 2}$.

Throughout this paper we shall assume that

(A1) $A(x)=\left(a_{i j}(x)\right)_{N \times N}$ is a real symmetric positive definite matrix function with $a_{i j} \in \mathbf{C}_{l o c}^{1+\nu}\left(\Omega\left(r_{0}\right), \mathbb{R}\right)$ for all $i, j, \nu \in(0,1)$;

(A1) $b_{i} \in \mathbf{C}_{l o c}^{\nu}\left(\Omega\left(r_{0}\right), \mathbb{R}\right)$ for all $i$;

(A1) $C \in \mathbf{C}_{l o c}^{\nu}\left(\Omega\left(r_{0}\right) \times \mathbb{R}, \mathbb{R}\right)$ with $C(x,-y)=-C(x, y)$ for all $x \in \mathbb{R}^{N}$ and $y \in \mathbb{R}$. Suppose that there exist functions $c \in \mathbf{C}_{l o c}^{\nu}\left(\Omega\left(r_{0}\right), \mathbb{R}\right)$ and $f \in \mathbf{C}(\mathbb{R}, \mathbb{R}) \cup \mathbf{C}^{1}(\mathbb{R}-\{0\}, \mathbb{R})$ with $y f(y)>0$ and $f^{\prime}(y) \geq k>0$

Received February 16, 2009.

2000 Mathematics Subject Classification. 35B05, 35J15, 35J60.

Key words and phrases. oscillation, annulus criteria, elliptic equations, second order, damped.

Research partially supported by the NSF of Guangdong Province under Grant 8451063101000730 . 
whenever $y \neq 0$ such that

$$
C(x, y) \geq c(x) f(y) \text { for all } x \in \Omega\left(r_{0}\right), y>0 .
$$

As usual, a function $y \in \mathbf{C}_{l o c}^{1+\nu}\left(\Omega\left(r_{0}\right), \mathbb{R}\right)$ with the property $a_{i j} D_{j} y \in \mathbf{C}_{l o c}^{1+\nu}$ $\left(\Omega\left(r_{0}\right), \mathbb{R}\right)$ is said to be a solution (classical solution) of Eq.(1.1) in $\Omega\left(r_{0}\right)$ if $y(x)$ satisfies Eq.(1.1) for all $x \in \Omega\left(r_{0}\right)$. For existence of solutions of Eq.(1.1), we refer the reader to the monograph [1]. We restrict our attention only to the nontrivial solution $y(x)$ of Eq.(1.1), i.e., $\sup \{|y(x)|:|x|>r\}>0$ for any $r \geq r_{0}$. A nontrivial solution $y(x)$ of Eq.(1.1) is called oscillatory in $\Omega\left(r_{0}\right)$ if the set $\left\{x \in \Omega\left(r_{0}\right): y(x)=0\right\}$ is unbounded; otherwise it is said to be nonoscillatory in $\Omega\left(r_{0}\right)$. Eq.(1.1) is oscillatory if all of its solutions are oscillatory in $\Omega\left(r_{0}\right)$.

Equation (1.1) is important for applications in physics, biology and glaciology, etc., see [1]. In the qualitative theory of nonlinear PDE, one of the important themes is to determine whether or not solutions of the equation under consideration are oscillatory. Since the pioneering work of Noussair and Swanson [7], there have been extensive investigations on oscillation for second order undamped elliptic equations. Swanson [9] summarized the oscillation results for undamped semilinear elliptic equations up to 1979. Recently, oscillation of elliptic equations with damping has become an important area of research due to the fact that such equations arises in real lift problems [1]. We refer the reader to the papers $[5,6,11-16,18]$ and the references cites therein.

In papers $[11-16,18]$, authors, starting with integration of the Riccati equation over spheres in $\mathbb{R}^{N}$ centered in the origin, covert the $N$-dimensional problem into a problem in one variable and then employ the integral averaging technique developed by Philos [8] to establish oscillation criteria for the following equation

$$
\sum_{i, j=1}^{N} D_{i}\left[a_{i j}(x) D_{j} y\right]+\sum_{i=1}^{N} b_{i}(x) D_{i} y+c(x) f(y)=0 .
$$

But, in this way, the oscillation criteria contained therein not only neglect the technical problems for high dimension space, but also deal with the oscillation of Eq.(1.2) only for the case that the mean value of the potential function $c(x)$ over the sphere centered in the origin is "sufficiently large".

On the other hand, in those papers [5, 6, 11-15], the conditions in terms of the coefficient involving integral averages over the whole exterior domain $\Omega\left(r_{0}\right)$. It is difficult to apply them to the case when $c(x)$ has "bad" behavior on a big part of $\Omega\left(r_{0}\right)$, e.g., when $\int_{\Omega\left(r_{0}\right)} c(x) d x=-\infty$. As we know, for ordinary differential equations, oscillation is an interval property, namely, it is more reasonable to investigate solutions on an infinite set of bounded intervals. The problem is therefore to find oscillation criteria which use only the information about the involved functions on these intervals, outside of these intervals the behavior of the functions is irrelevant. Such type of oscillation criteria are refereed to as the interval oscillation criteria. Using this thought, in 1999, Kong [3] employed 
the technique developed by Philos [8] and presented several beautiful interval oscillation criteria. Recently, Kong's results were extended to a general class of second order nonlinear differential equations with nonlinear damping and second order matrix differential systems by Tiryaki and Zafer [10] and Kong [4], respectively, see also [17]. Therefore, it is natural to ask if it is possible to extend Kong's theorem to Eq.(1.1). An affirmative answer to this problem was given by $\mathrm{Xu}$ and Xing [16] and Zhuang and Yao [18], independently, who obtained Kong-type oscillation criteria for Eq.(1.2). However, the oscillation theorems require additional assumptions which are imposed the differentiability on $b_{i}(x)$ for all $i$.

Inspired by the ideas of Kong [3] and Mařík [5], in this paper, we prefer an advanced approach than that one used in papers $[6,7,11-16,18]$, that is not based on conversion of Eq.(1.1) into an ordinary differential inequality. Following this technique, we extend Kong's results [3] to Eq.(1.1) and establish annulus oscillation criteria for Eq.(1.1) by dropping the differentiability on $b_{i}(x)$. The results obtain here only base on the information on a sequence of annuluses of $\Omega\left(r_{0}\right)$, rather then on the whole exterior domain $\Omega\left(r_{0}\right)$. In particular, our results can be applied to the extreme case such as $\int_{\Omega\left(r_{0}\right)} c(x) d x=-\infty$. Our methodology is somewhat different from that of previous authors. We believe in some sense that our method is are more natural for studying for oscillation of PDE.

\section{Annulus oscillation}

Before proceeding, we set some notation and terminology that will be used throughout this paper. Let

$$
\begin{aligned}
& S(a)=\left\{x \in \mathbb{R}^{N}:|x|=a\right\}, \\
& D=\left\{(r, s) \in \mathbb{R}^{2}, r \geq s>r_{0}\right\}, \\
& \Omega(a, b)=\left\{x \in \mathbb{R}^{N}: a<|x|<b\right\}, \\
& \Omega[a, b]=\left\{x \in \mathbb{R}^{N}: a \leq|x| \leq b\right\}, \\
& \nabla=\left(D_{1}, D_{2}, \ldots, D_{N}\right), \\
& B(x)=\left(b_{1}(x), b_{2}(x), \ldots, b_{N}(x)\right), \\
& A^{-1}(x) \text { denotes the inverse of } A(x), \\
& \langle,\rangle \text { is the usual scalar produce in } \mathbb{R}^{N}, \\
& d \sigma \text { represents the integral element of the sphere } S(|x|), \\
& \nu(x)=x /|x| \text { denotes the outside normal unit vector to the sphere } S(|x|), \\
& \lambda_{\text {max }}(x) \text { denotes the largest eigenvalue of the matrix } A(x) . \\
& \text { Clearly, it follows from (A1) that } \lambda_{\text {max }}(x)>0 \text { for all } x \in \Omega\left(r_{0}\right) .
\end{aligned}
$$

Definition 2.1. The function $H_{1} \in \mathbf{C}(D,[0, \infty))$ is said to belong to the function class $\mathcal{H}_{1}$, defined by $H_{1} \in \mathcal{H}_{1}$, if 
(1) $H_{1}(r, s)=0$ if and only if $r=s$;

(2) The partial derivative $\partial H_{1}(r, s) / \partial r$ exists. Define

$$
h_{1}(r, s)=\frac{1}{H_{1}(r, s)} \frac{\partial H_{1}(r, s)}{\partial r} \quad \text { for }(r, s) \in D, r \neq s,
$$

the function $h_{1}$ is locally integrable on each compact subset in $D$.

Definition 2.2. The function $H_{2} \in \mathbf{C}(D,[0, \infty))$ is said to the function class $\mathcal{H}_{2}$, defined by $H_{2} \in \mathcal{H}_{2}$, if

(3) $H_{2}(r, s)=0$ if and only if $r=s$;

(4) The partial derivative $\partial H_{2}(r, s) / \partial s$ exists. Denote

$$
h_{2}(r, s)=-\frac{1}{H_{2}(r, s)} \frac{\partial H_{2}(r, s)}{\partial s} \text { for }(r, s) \in D, r \neq s,
$$

the function $h_{2}$ is locally integrable on each compact subset in $D$.

For convenience, we define the function notations as follows:

$$
\begin{aligned}
& \Theta_{1}(|x|, a)=h_{1}(|x|, a) \nu(x)-B(x) A^{-1}(x)+\frac{\nabla \rho(x)}{\rho(x)}, \\
& \Theta_{2}(b,|x|)=h_{2}(b,|x|) \nu(x)+B(x) A^{-1}(x)-\frac{\nabla \rho(x)}{\rho(x)}, \\
& \lambda(r)=\int_{S(r)} \rho(x) \lambda_{\max }(x) d \sigma, \quad \Lambda(r)=\int_{r_{0}}^{r} \frac{d s}{\lambda(s)}, \\
& \breve{c}(x)=c(x)-\frac{1}{4 k \varepsilon} \lambda_{\max }(x)\left|B(x) A^{-1}(x)-\frac{\nabla \rho(x)}{\rho(x)}\right|^{2},
\end{aligned}
$$

where $h_{1}, h_{2} \in \mathbf{C}(D, \mathbb{R}), \rho \in \mathbf{C}^{1}\left(\Omega\left(r_{0}\right), \mathbb{R}^{+}\right)$and $\varepsilon \in(0,1)$.

To prove our results, we need several preparatory considerations.

Lemma 2.1. Let $X, Y \in \mathbb{R}^{N}$. Then the following elementary inequality hold.

$$
\langle X, Y\rangle \leq \kappa|X|^{2}+\frac{1}{4 \kappa}|Y|^{2} \quad \text { for any } \kappa>0 .
$$

The proof is straightforward and omitted.

Lemma 2.2. Let $A_{0} \in \mathbf{C}\left(\Omega\left(r_{0}\right), \mathbb{R}\right), A_{1} \in \mathbf{C}\left(\Omega\left(r_{0}\right), \mathbb{R}^{N}\right), A_{2} \in \mathbf{C}\left(\Omega\left(r_{0}\right), \mathbb{R}^{+}\right)$ and $z \in \mathbf{C}\left(\Omega\left(r_{0}\right), \mathbb{R}^{N}\right)$. If there exists $(a, b) \subset\left[r_{0}, \infty\right)$ such that

$$
\operatorname{div}(z(x)) \leq-A_{0}(x)-\left\langle A_{1}(x), z(x)\right\rangle-A_{2}(x)|z(x)|^{2}, \quad x \in \Omega(a, b),
$$

then for any $\ell \in(a, b)$ and any $H_{i} \in \mathcal{H}_{i}, i=1,2$,

$$
\begin{aligned}
& \frac{1}{H_{1}(\ell, a)} \int_{\Omega[a, \ell]} H_{1}(|x|, a)\left[A_{0}(x)-\frac{1}{4 A_{2}(x)}\left|\Pi_{1}(|x|, a)\right|^{2}\right] d x \\
& +\frac{1}{H_{2}(b, \ell)} \int_{\Omega[\ell, b]} H_{2}(b,|x|)\left[A_{0}(x)-\frac{1}{4 A_{2}(x)}\left|\Pi_{2}(b,|x|)\right|^{2}\right] d x \leq 0,
\end{aligned}
$$


where

$$
\Pi_{1}(|x|, a)=h_{1}(|x|, a) \nu(x)-A_{1}(x), \quad \Pi_{2}(b,|x|)=h_{2}(b,|x|) \nu(x)+A_{1}(x) .
$$

Proof. Multiplying both sides of $(2.1)$ by $H_{1}(|x|, r)$ and integrating over the annulus $\Omega[r, \ell]$ for $r \in(a, \ell]$, we get

$$
\begin{aligned}
& \int_{\Omega[r, \ell]} H_{1}(|x|, r) A_{0}(x) d x \\
\leq & -\int_{r}^{\ell} H_{1}(s, r) \int_{S(s)} \operatorname{div}(z(x)) d \sigma d s-\int_{\Omega[r, \ell]} H_{1}(|x|, r)\left\langle A_{1}(x), w(x)\right\rangle d x \\
& -\int_{\Omega[r, \ell]} H_{1}(|x|, r) A_{2}(x)|z(x)|^{2} d x .
\end{aligned}
$$

Integration by parts in the first integral of the right side of the above, and by Gauss formula and the definition of the function $h_{1}$ give that

$$
\begin{aligned}
& \int_{\Omega[r, \ell]} H_{1}(|x|, r) A_{0}(x) d x \\
\leq & -H(\ell, r) \int_{S(\ell)}\langle z(x), \nu(x)\rangle d \sigma+\int_{\Omega[r, \ell]} H_{1}(|x|, r)\left\langle z(x), \Pi_{1}(|x|, r)\right\rangle d x \\
& -\int_{\Omega[r, \ell]} H_{1}(|x|, r) A_{2}(x)|z(x)|^{2} d x .
\end{aligned}
$$

By Lemma 2.1,

$$
\left\langle z(x), \Pi_{1}(|x|, r)\right\rangle \leq A_{2}(x)|z(x)|^{2}+\frac{1}{4 A_{2}(x)}\left|\Pi_{1}(|x|, r)\right|^{2} .
$$

Combining the above and (2.3), then letting $r \rightarrow a^{+}$and dividing the resulting inequality through by $H_{1}(\ell, a)$, one has

$$
\begin{aligned}
& \frac{1}{H_{1}(\ell, a)} \int_{\Omega[a, \ell]} H_{1}(|x|, a)\left[A_{0}(x)-\frac{1}{4 A_{2}(x)}\left|\Pi_{1}(|x|, a)\right|^{2}\right] d x \\
\leq & -\int_{S(\ell)}\langle z(x), \nu(x)\rangle d \sigma .
\end{aligned}
$$

Similarly, if the both sides of (2.1) is multiplied by $H_{2}(r,|x|)$ and then integrated over $\Omega[\ell, r]$ for $r \in[\ell, b)$, then proceeding as the proof of (2.4), we can obtain

$$
\frac{1}{H_{1}(b, \ell)} \int_{\Omega[\ell, b]} H_{2}(b,|x|)\left[A_{0}(x)-\frac{1}{4 A_{2}(x)}\left|\Pi_{2}(b,|x|)\right|^{2}\right] d x \leq \int_{S(\ell)}\langle z(x), \nu(x)\rangle d \sigma .
$$

Adding (2.4) and (2.5), we have (2.2).

Lemma 2.3 ([12]). Let $y=y(x)$ be a nonoscillatory solution of Eq.(1.1) and $\rho \in \mathbf{C}^{1}\left(\Omega\left(r_{0}\right), \mathbb{R}^{+}\right)$. Then $N$-dimensional vector function $w(x)$ defined by

$$
w(x)=\frac{1}{f(y)}(A \nabla y)(x)
$$


satisfies the partial Riccati inequality

$$
\begin{aligned}
\operatorname{div}(\rho(x) w(x)) \leq & -\rho(x) c(x)-\left\langle\rho(x) B(x) A^{-1}(x)-\nabla \rho(x), w(x)\right\rangle \\
& -k \rho(x) \lambda_{\max }^{-1}(x)|w(x)|^{2} .
\end{aligned}
$$

Lemma 2.4. Suppose that for any interval $(a, b) \subset\left[r_{0}, \infty\right)$, there exist some $\ell \in(a, b)$ and functions $H_{i} \in \mathcal{H}_{i}, i=1,2, \rho \in \mathbf{C}^{1}\left(\Omega\left(r_{0}\right), \mathbb{R}^{+}\right)$such that

$$
\begin{aligned}
& \frac{1}{H_{1}(\ell, a)} \int_{\Omega[a, \ell]} H_{1}(|x|, a) \rho(x)\left[c(x)-\frac{1}{4 k} \lambda_{\max }(x)\left|\Theta_{1}(|x|, a)\right|^{2}\right] d x \\
& +\frac{1}{H_{2}(b, \ell)} \int_{\Omega[\ell, b]} H_{2}(b,|x|) \rho(x)\left[c(x)-\frac{1}{4 k} \lambda_{\max }(x)\left|\Theta_{2}(b,|x|)\right|^{2}\right] d x>0 .
\end{aligned}
$$

Then every solution of Eq.(1.1) has at least one zero in the annulus $\Omega(a, b)$.

Proof. Otherwise, $y(x) \neq 0$ for all $x \in \Omega(a, b)$. Defined $w(x)$ by $(2.6)$, by Lemma 2.3, (2.7) holds. Comparing inequalities (2.1) and (2.7) we identify that

$$
\begin{aligned}
& z(x)=\rho(x) w(x), A_{0}(x)=\rho(x) c(x), \\
& A_{1}(x)=B A^{-1}-\frac{\nabla \rho(x)}{\rho(x)}, A_{2}(x)=\frac{k}{\rho(x) \lambda_{\max }(x)} .
\end{aligned}
$$

Applying Lemma 2.2 to (2.7) we see that the inequality (2.8) fails to hold.

If the conditions of Lemma 2.4 holds for a sequence $\left\{\Omega\left(a_{i}, b_{i}\right)\right\}$ of the annuluses such that $\lim _{i \rightarrow \infty} a_{i}=\infty$, then we may conclude that Eq.(1.1) is oscillatory. Hence, we have the following theorem.

Theorem 2.1. If for each $T \geq r_{0}$, there exists an interval $(a, b) \subset[T, \infty)$ for which the conditions of Lemma 2.4 are satisfied, then Eq.(1.1) is oscillatory.

The following theorem is an immediate result from Theorem 2.1.

Theorem 2.2. If there exist functions $H_{i} \in \mathcal{H}_{i}, i=1,2$, and $\rho \in \mathbf{C}^{1}\left(\Omega\left(r_{0}\right), \mathbb{R}^{+}\right)$ such that for each $l \geq r_{0}$,

$$
\limsup _{r \rightarrow \infty} \frac{\int_{\Omega[l, r]} H_{1}(|x|, l) \rho(x) c(x) d x}{\int_{\Omega[l, r]} H_{1}(|x|, l) \rho(x) \lambda_{\max }(x)\left|\Theta_{1}(|x|, l)\right|^{2} d x}=\infty
$$

and

$$
\limsup _{r \rightarrow \infty} \frac{\int_{\Omega[l, r]} H_{2}(r,|x|) \rho(x) c(x) d x}{\int_{\Omega[l, r]} H_{2}(r,|x|) \rho(x) \lambda_{\max }(x)\left|\Theta_{2}(r,|x|)\right|^{2} d x}=\infty,
$$

then Eq.(1.1) is oscillatory.

Proof. For any $T \geq r_{0}$, let $a=T$. In (2.9) we choose $l=a$. Then there exists $\ell>a$ such that

$$
\int_{\Omega[a, \ell]} H_{1}(|x|, a) \rho(x) c(x) d x>\frac{1}{4 k} \int_{\Omega[a, \ell]} H_{1}(|x|, a) \rho(x) \lambda_{\max }(x)\left|\Theta_{1}(|x|, a)\right|^{2} d x .
$$


In $(2.10)$ we choose $l=\ell$. Then there exists $b>\ell$ such that

$$
\int_{\Omega[\ell, b]} H_{2}(b,|x|) \rho(x) c(x) d x>\frac{1}{4 k} \int_{\Omega[\ell, b]} H_{2}(b,|x|) \rho(x) \lambda_{\max }(x)\left|\Theta_{2}(b,|x|)\right|^{2} d x .
$$

Combining (2.11) and (2.12) we obtain (2.8) holds. The conclusion thus comes from Theorem 2.1.

The following results is also a consequence of Theorem 2.1 whose proof is similar to that of Theorem 2.2.

Theorem 2.3. If there exist functions $H_{i} \in \mathcal{H}_{i}, i=1,2$, and $\rho \in \mathbf{C}^{1}\left(\Omega\left(r_{0}\right), \mathbb{R}^{+}\right)$ such that for each $l \geq r_{0}$,

$$
\limsup _{r \rightarrow \infty} \int_{\Omega[l, r]} H_{1}(|x|, l) \rho(x)\left[c(x)-\frac{1}{4 k} \lambda_{\max }(x)\left|\Theta_{1}(|x|, l)\right|^{2}\right] d x>0
$$

and

(2.14) $\quad \limsup _{r \rightarrow \infty} \int_{\Omega[l, r]} H_{2}(r,|x|) \rho(x)\left[c(x)-\frac{1}{4 k} \lambda_{\max }(x)\left|\Theta_{2}(r,|x|)\right|^{2}\right] d x>0$,

then Eq.(1.1) is oscillatory.

Remark 2.1. If $N=1, b_{i}(x)=0$ for all $i$ and $\rho(x)=1$, then Theorems 2.1 and 2.3 reduce Theorem 2.1 and Corollary 2.4, respectively, given in [3]. On the other hand, if $N=1$, then Theorem 2.1 reduces Theorem 1 in [17].

Remark 2.2. For Eq.(1.2), Theorems 2.1 and 2.3 improve Theorems 2.1 and 2.2 in [16], and Theorems 2.2 and 2.3 in [18].

Next, we define

$$
H_{1}(r, s)=[\Lambda(r)-\Lambda(s)]^{\alpha}, \quad H_{2}(r, s)=[\Lambda(r)-\Lambda(s)]^{\beta}, \quad r \geq s \geq r_{0},
$$

where $\alpha, \beta>1$ are constants.

By Theorems 2.2 and 2.3, we have the following oscillation criteria.

Corollary 2.1. If there exist a function $\rho \in \mathbf{C}^{1}\left(\Omega\left(r_{0}\right), \mathbb{R}\right)$ with

$$
\nabla \rho(x)=\rho(x) B(x) A^{-1}(x)
$$

and some $\alpha, \beta>1$ such that for each $l \geq r_{0}$,

$$
\limsup _{r \rightarrow \infty} \frac{1}{[\Lambda(r)-\Lambda(l)]^{\alpha-1}} \int_{\Omega[l, r]}[\Lambda(|x|)-\Lambda(l)]^{\alpha} \rho(x) c(x) d x=\infty
$$

and

$$
\limsup _{r \rightarrow \infty} \frac{1}{[\Lambda(r)-\Lambda(l)]^{\beta-1}} \int_{\Omega[l, r]}[\Lambda(r)-\Lambda(|x|)]^{\beta} \rho(x) c(x) d x=\infty,
$$

then Eq.(1.1) is oscillatory. 
Proof. Taking $H_{1}(r, s)$ and $H_{2}(r, s)$ as in (2.15), we get

$$
\Theta_{1}(|x|, l)=\frac{\alpha}{\Lambda(|x|)-\Lambda(l)} \frac{1}{\lambda(|x|)} \nu(x), \quad \Theta_{2}(r,|x|)=\frac{\beta}{\Lambda(r)-\Lambda(|x|)} \frac{1}{\lambda(|x|)} \nu(x) .
$$

Note that

$$
\begin{aligned}
& \int_{\Omega[l, r]} H_{1}(|x|, l) \rho(x) \lambda_{\max }(x)\left|\Theta_{1}(|x|, l)\right|^{2} d x \\
= & \int_{l}^{r} H_{1}(s, l)\left|\Theta_{1}(s, l)\right|^{2} \int_{S(s)} \rho(x) \lambda_{\max }(x) d \sigma d s \\
= & \frac{\alpha^{2}}{\alpha-1}[\Lambda(r)-\Lambda(l)]^{\alpha-1}
\end{aligned}
$$

and

$$
\begin{aligned}
& \int_{\Omega[l, r]} H_{2}(r,|x|) \rho(x) \lambda_{\max }(x)\left|\Theta_{2}(r,|x|)\right|^{2} d x \\
= & \int_{l}^{r} H_{2}(r, s)\left|\Theta_{2}(r, s)\right|^{2} \int_{S(s)} \rho(x) \lambda_{\max }(x) d \sigma d s \\
= & \frac{\beta^{2}}{\beta-1}[\Lambda(r)-\Lambda(l)]^{\beta-1} .
\end{aligned}
$$

Combining (2.16) and (2.18), we obtain (2.9). Similarly, (2.17) and (2.19) implies that (2.10) holds. By Theorem 2.2, Eq.(1.1) is oscillatory.

Corollary 2.2. If there exist a function $\rho \in \mathbf{C}^{1}\left(\Omega\left(r_{0}\right), \mathbb{R}\right)$ with

$$
\nabla \rho(x)=\rho(x) B(x) A^{-1}(x)
$$

satisfying $\lim _{r \rightarrow \infty} \Lambda(r)=\infty$ and some $\alpha, \beta>1$ such that for each $l \geq r_{0}$,

$$
\limsup _{r \rightarrow \infty} \frac{1}{\Lambda^{\alpha-1}(r)} \int_{\Omega[l, r]}[\Lambda(|x|)-\Lambda(l)]^{\alpha} \rho(x) c(x) d x>\frac{\alpha^{2}}{4 k(\alpha-1)}
$$

and

$$
\limsup _{r \rightarrow \infty} \frac{1}{\Lambda^{\beta-1}(r)} \int_{\Omega[l, r]}[\Lambda(r)-\Lambda(|x|)]^{\beta} \rho(x) c(x) d x>\frac{\beta^{2}}{4 k(\beta-1)},
$$

then Eq.(1.1) is oscillatory.

Proof. Similar to the proof of Corollary 2.1, we get (2.18) and (2.19) hold. The fact that $\lim _{r \rightarrow \infty} \Lambda(r)=\infty$ follows that

$(2.22) \quad \limsup _{r \rightarrow \infty} \frac{1}{\Lambda^{\alpha-1}(r)} \int_{\Omega[l, r]} H_{1}(|x|, l) \rho(x) \lambda_{\max }(x)\left|\Theta_{1}(|x|, l)\right|^{2} d x=\frac{\alpha^{2}}{\alpha-1}$

and

(2.23) $\limsup _{r \rightarrow \infty} \frac{1}{\Lambda^{\alpha-1}(r)} \int_{\Omega[l, r]} H_{2}(r,|x|) \rho(x) \lambda_{\max }(x)\left|\Theta_{2}(r,|x|)\right|^{2} d x=\frac{\beta^{2}}{\beta-1}$. 
Therefore, (2.20) and (2.22) implies that (2.13) holds. (2.21) and (2.23) follows that (2.14) holds. By Theorem 2.3, Eq.(1.1) is oscillatory.

Corollary 2.3. If there exist a function $\rho \in \mathbf{C}^{1}\left(\Omega\left(r_{0}\right), \mathbb{R}\right)$ with

$$
\nabla \rho(x)=\rho(x) B(x) A^{-1}(x)
$$

satisfying $\lim _{r \rightarrow \infty} \Lambda(r)=\infty$ and some $u>1 /(4 k)$ such that

$$
\int_{S(r)} \rho(x) c(x) d \sigma \geq \frac{u}{\Lambda^{2}(r)} \frac{1}{\lambda(r)},
$$

then Eq.(1.1) is oscillatory.

Proof. Note that

$$
\begin{aligned}
& \limsup _{r \rightarrow \infty} \frac{1}{\Lambda^{\alpha-1}(r)} \int_{\Omega[l, r]}[\Lambda(|x|)-\Lambda(l)]^{\alpha} \rho(x) c(x) d x \\
= & \limsup _{r \rightarrow \infty} \frac{1}{\Lambda^{\alpha-1}(r)} \int_{l}^{r}[\Lambda(s)-\Lambda(l)]^{\alpha} \int_{S(s)} \rho(x) c(x) d \sigma d s \quad \text { (by (2.24)) } \\
\geq & \limsup _{r \rightarrow \infty} \frac{1}{\Lambda^{\alpha-1}(r)} \int_{l}^{r}[\Lambda(s)-\Lambda(l)]^{\alpha} \frac{u}{\Lambda^{2}(s) \lambda(s)} d s \quad \text { (by L'Hopital's rule) } \\
= & \frac{u}{\alpha-1} \limsup \frac{[\Lambda(r)-\Lambda(l)]^{\alpha}}{\Lambda^{\alpha}(r)}=\frac{u}{\alpha-1} .
\end{aligned}
$$

Consequently, for any $u>1 /(4 k)$, there exists a constant $\alpha>1$ such that

$$
\frac{u}{\alpha-1}>\frac{\alpha^{2}}{4 k(\alpha-1)} \text {. }
$$

This means that (2.20) holds. 41]

On the other hand, it follows from the fundamental inequality $[2$, Theorem

$$
(r-s)^{\beta} \geq r^{\beta}-\beta s r^{\beta-1} \quad \text { for } r>s \geq r_{0},
$$

that

$$
[\Lambda(r)-\Lambda(s)]^{\beta} \geq \Lambda^{\beta}(r)-\beta \Lambda(s) \Lambda^{\beta-1}(r) \text { for } r \geq s \geq r_{0} .
$$

Therefore,

$$
\begin{aligned}
& \limsup _{r \rightarrow \infty} \frac{1}{\Lambda^{\beta-1}(r)} \int_{\Omega[l, r]}[\Lambda(r)-\Lambda(s)]^{\beta} \rho(x) c(x) d x \\
= & \limsup _{r \rightarrow \infty} \frac{1}{\Lambda^{\beta-1}(r)} \int_{l}^{r}[\Lambda(r)-\Lambda(s)]^{\beta} \int_{S(s)} \rho(x) c(x) d \sigma d x \\
\geq & \limsup _{r \rightarrow \infty} \frac{1}{R^{\beta-1}(r)} \int_{l}^{r}\left[\Lambda^{\beta}(r)-\beta \Lambda(s) \Lambda^{\beta-1}(r)\right] \frac{u}{\Lambda^{2}(s)} \frac{1}{\lambda(s)} d s \\
= & u \limsup _{r \rightarrow \infty}\left(\frac{\Lambda(r)}{\Lambda(l)}-\beta \ln \frac{\Lambda(r)}{\Lambda(l)}-1\right)=\infty .
\end{aligned}
$$


Hence, for any $u>0$ and $\beta>1$, we have

$$
\limsup _{r \rightarrow \infty} \frac{1}{\Lambda^{\beta-1}(r)} \int_{\Omega[l, r]}[\Lambda(r)-\Lambda(|x|)]^{\beta} \rho(x) c(x) d x=\infty>\frac{\beta^{2}}{4 k(\beta-1)},
$$

i.e., (2.21) holds. Applying Corollary 2.2, we find Eq.(1.1) is oscillatory.

The remaining part of this section, using Lemma 2.1 for (2.7), we present another set of oscillation results which different from Theorems 2.1, 2.2, 2.3 and Corollaries 2.1, 2.2, 2.3. For this, we first establish the following lemma corresponding to Lemma 2.4 .

Lemma 2.5. Suppose that for any interval $(a, b) \subset\left[r_{0}, \infty\right)$, there exist some $\ell \in(a, b)$ and functions $H_{i} \in \mathcal{H}_{i}, i=1,2, \rho \in \mathbf{C}^{1}\left(\Omega\left(r_{0}\right), \mathbb{R}^{+}\right)$such that

$$
\begin{aligned}
& \frac{1}{H_{1}(\ell, a)} \int_{\Omega[a, \ell]} H_{1}(|x|, a) \rho(x)\left[\breve{c}(x)-\frac{1}{4 k(1-\varepsilon)} \lambda_{\max }(x)\left|h_{1}(|x|, a)\right|^{2}\right] d x \\
& +\frac{1}{H_{2}(b, \ell)} \int_{\Omega[\ell, b]} H_{2}(b,|x|) \rho(x)\left[\breve{c}(x)-\frac{1}{4 k(1-\varepsilon)} \lambda_{\max }(x)\left|h_{2}(b,|x|)\right|^{2}\right] d x>0,
\end{aligned}
$$

where $\varepsilon \in(0,1)$ is a constant if $\rho(x) B(x) A^{-1}(x) \neq \nabla \rho(x)$, and $\varepsilon=0$ if $\rho(x) B(x) A^{-1}(x)=\nabla \rho(x)$. Then every solution of Eq.(1.1) has at least one zero in the annulus $\Omega(a, b)$.

Proof. Otherwise, $y(x) \neq 0$ for all $x \in \Omega(a, b)$. Defined $w(x)$ by (2.6), by Lemma 2.3, (2.7) holds.

Case 1. If $\rho(x) B(x) A^{-1}(x)-\nabla \rho(x) \neq 0$, by Lemma 2.1, then

$$
\begin{aligned}
& \left\langle B A^{-1}-\frac{\nabla \rho(x)}{\rho(x)}, w(x)\right\rangle \\
\leq & \frac{1}{4 k \varepsilon} \lambda_{\max }(x)\left|B(x) A^{-1}(x)-\frac{\nabla \rho(x)}{\rho(x)}\right|^{2}+k \varepsilon \lambda_{\max }^{-1}(x)|w(x)|^{2} .
\end{aligned}
$$

Combining the above and (2.7), one has

$$
\operatorname{div}(\rho(x) w(x)) \leq-\rho(x) \breve{c}(x)-k(1-\varepsilon) \lambda_{\max }^{-1}(x)|w(x)|^{2} .
$$

It follows from the last inequality that (2.1) holds with

$$
z(x)=\rho(x) w(x), A_{0}(x)=\rho(x) \breve{c}(x), A_{1}(x)=0, A_{2}(x)=\frac{k(1-\varepsilon)}{\rho(x) \lambda_{\max }(x)} .
$$

Applying Lemma 2.2 to (2.26) we see that inequality (2.25) fails to hold.

Case 2. If $\rho(x) B(x) A^{-1}(x)-\nabla \rho(x)=0$, then (2.7) reduces

$$
\operatorname{div}(\rho(x) w(x)) \leq-\rho(x) c(x)-k \rho(x) \lambda_{\max }^{-1}(x)|w(x)|^{2} .
$$

By Lemma 2.2, we also get a desired contradiction to (2.25) for $\varepsilon=0$.

The following results are the counterparts of Theorems 2.1, 2.2, 2.3 and Corollaries 2.1, 2.2, 2.3, which can be proved in a similar manner. 
Theorem 2.4. If for each $T \geq r_{0}$, there exists an interval $(a, b) \subset[T, \infty)$ for which the conditions of Lemma 2.5 are satisfied, then Eq.(1.1) is oscillatory.

Theorem 2.5. If there exist functions $H_{i} \in \mathcal{H}_{i}, i=1,2$, and $\rho \in \mathbf{C}^{1}\left(\Omega\left(r_{0}\right), \mathbb{R}^{+}\right)$ such that for each $l \geq r_{0}$,

$$
\limsup _{r \rightarrow \infty} \frac{\int_{\Omega[l, r]} H_{1}(|x|, l) \rho(x) \breve{c}(x) d x}{\int_{\Omega[l, r]} H_{1}(|x|, l) \rho(x) \lambda_{\max }(x)\left|h_{1}(|x|, l)\right|^{2} d x}=\infty
$$

and

$$
\limsup _{r \rightarrow \infty} \frac{\int_{\Omega[l, r]} H_{2}(r,|x|) \rho(x) \breve{c}(x) d x}{\int_{\Omega[l, r]} H_{2}(r,|x|) \rho(x) \lambda_{\max }(x)\left|h_{2}(r,|x|)\right|^{2} d x}=\infty,
$$

then Eq.(1.1) is oscillatory.

Theorem 2.6. If there exist functions $H_{i} \in \mathcal{H}_{i}, i=1,2$, and $\rho \in \mathbf{C}^{1}\left(\Omega\left(r_{0}\right), \mathbb{R}^{+}\right)$ such that for each $l \geq r_{0}$,

(2.29) $\limsup _{r \rightarrow \infty} \int_{\Omega[l, r]} H_{1}(|x|, l) \rho(x)\left[\breve{c}(x)-\frac{1}{4 k(1-\varepsilon)} \lambda_{\max }(x)\left|h_{1}(|x|, l)\right|^{2}\right] d x>0$

and

$$
\limsup _{r \rightarrow \infty} \int_{\Omega[l, r]} H_{2}(r,|x|) \rho(x)\left[\breve{c}(x)-\frac{1}{4 k(1-\varepsilon)} \lambda_{\max }(x)\left|h_{2}(r,|x|)\right|^{2}\right] d x>0,
$$

where $\varepsilon$ is same as in Lemma 2.5, then Eq.(1.1) is oscillatory.

Corollary 2.4. If there exist a function $\rho \in \mathbf{C}^{1}\left(\Omega\left(r_{0}\right), \mathbb{R}\right)$ and some $\alpha, \beta>1$ such that for each $l \geq r_{0}$,

$$
\limsup _{r \rightarrow \infty} \frac{1}{[\Lambda(r)-\Lambda(l)]^{\alpha-1}} \int_{\Omega[l, r]}[\Lambda(|x|)-\Lambda(l)]^{\alpha} \rho(x) \breve{c}(x) d x=\infty
$$

and

$$
\limsup _{r \rightarrow \infty} \frac{1}{[\Lambda(r)-\Lambda(l)]^{\beta-1}} \int_{\Omega[l, r]}[\Lambda(r)-\Lambda(|x|)]^{\beta} \rho(x) \breve{c}(x) d x=\infty,
$$

then Eq.(1.1) is oscillatory.

Corollary 2.5. If there exist a function $\rho \in \mathbf{C}^{1}\left(\Omega\left(r_{0}\right), \mathbb{R}\right)$ satisfying $\lim _{r \rightarrow \infty} \Lambda(r)$ $=\infty$, and some $\alpha, \beta>1$ such that for each $l \geq r_{0}$,

(2.33) $\limsup _{r \rightarrow \infty} \frac{1}{\Lambda^{\alpha-1}(r)} \int_{\Omega[l, r]}[\Lambda(|x|)-\Lambda(l)]^{\alpha} \rho(x) \breve{c}(x) d x>\frac{\alpha^{2}}{4 k(1-\varepsilon)(\alpha-1)}$

and

(2.34) $\limsup _{r \rightarrow \infty} \frac{1}{\Lambda^{\beta-1}(r)} \int_{\Omega[l, r]}[\Lambda(r)-\Lambda(|x|)]^{\beta} \rho(x) \breve{c}(x) d x>\frac{\beta^{2}}{4 k(1-\varepsilon)(\beta-1)}$,

where $\varepsilon$ is same as in Lemma 2.5, then Eq.(1.1) is oscillatory. 
Corollary 2.6. If there exist a function $\rho \in \mathbf{C}^{1}\left(\Omega\left(r_{0}\right), \mathbb{R}\right)$ satisfying $\lim _{r \rightarrow \infty} \Lambda(r)$ $=\infty$, and some $u>1 /(4 k)$ such that

$$
\int_{S(r)} \rho(x) \breve{c}(x) d \sigma \geq \frac{u}{\Lambda^{2}(r)} \frac{1}{\lambda(r)},
$$

then Eq.(1.1) is oscillatory.

Remark 2.3. We note that it is suffices to satisfy (2.25) in Theorem 2.4, which ensures a certain flexibility in applications. Clearly, if (2.25) is satisfied for some $\varepsilon_{0} \in(0,1)$, it shall also hold for any $\varepsilon \in\left(0, \varepsilon_{0}\right)$. This property for $\varepsilon$ also holds for Theorems 2.5, 2.6 and Corollaries 2.4, 2.5, 2.6.

Remark 2.4. $f(y)$ may not be differentiable or increasing, for example, $f(x)$ satisfies

$$
\frac{f(y)}{y} \geq \kappa>0 \text { for all } x \neq 0 .
$$

In this case, when $c(x) \geq 0$ for all $x \in \Omega\left(r_{0}\right)$ and $c(x)$ does not vanish eventually, define $w(x)$ by

$$
w(x)=\frac{1}{y}(A \nabla y)(x)
$$

following the slight modification of the proofs of the corresponding theorems given in this paper, we can obtain new set of oscillation criteria for Eq.(1.1). Here, we leave the statements and proofs of these results to the interested reader.

Remark 2.5. Note that the functions $H_{i}(i=1,2)$ play slightly different role here than those in $[3,4,10,16,17,18]$. The reason is that we wish to give simple formulas in our results.

Remark 2.6. The oscillation of Eq.(1.2) has been investigated by many authors. Our results, however, are still new because we drop the differentiability of the damped functions $b_{i}(x)$ for all $i$. The arbitrariness of function $\rho(x)$ and the classes of functions $\mathcal{H}_{i}, i=1,2$, provide more flexibilities for deriving new oscillation criteria. Though we have chosen $H_{i}, i=1,2$, defined by (2.15), there are interesting possibilities to apply our results, for instance, with

$$
H_{1}(r, s)=\left(\int_{s}^{r} \frac{d u}{\phi(u)}\right)^{\alpha} \text { and } H_{2}(r, s)=\left(\int_{s}^{r} \frac{d u}{\psi(u)}\right)^{\beta}, \quad(r, s) \in D,
$$

where $\alpha, \beta>1, \phi, \psi \in \mathbf{C}\left(\left[r_{0}, \infty\right), \mathbb{R}^{+}\right)$satisfy $\int_{r_{0}}^{\infty} d u / \phi(u)=\infty$ and $\int_{r_{0}}^{\infty} d u / \psi(u)$ $=\infty$.

Finally, we provide two examples to illustrate the main results.

Example 2.1. Consider the following elliptic equation (2.36)

$$
\frac{\partial}{\partial x_{1}}\left(\frac{1}{|x|} \frac{\partial y}{\partial x_{1}}\right)+\frac{\partial}{\partial x_{2}}\left(\frac{1}{|x|} \frac{\partial y}{\partial x_{2}}\right)+\frac{1}{|x|^{4}}\left(x_{1} \frac{\partial y}{\partial x_{1}}+x_{2} \frac{\partial y}{\partial x_{2}}\right)+c(x)\left(y+y^{3}\right)=0,
$$


where $x \in \Omega(1)$, and

$$
c(x)= \begin{cases}|x|-3 n, & 3 n \leq|x| \leq 3 n+1, \\ -|x|+3 n+2, & 3 n+1<|x| \leq 3 n+2, \\ c_{0}(x), & 3 n+2<|x| \leq 3 n+3,\end{cases}
$$

for $n \in\{1,2, \ldots\}$, where $c_{0} \in \mathbf{C}^{\nu}\left(\Omega\left(r_{0}\right), \mathbb{R}\right)$ which makes $c \in \mathbf{C}^{\nu}\left(\Omega\left(r_{0}\right), \mathbb{R}\right)$. It is worth mentioning that by a suitable choice of $c_{0}(x)$ we can make $\int_{\Omega\left(r_{0}\right)} c(x) d x=$ $-\infty$.

Take $H_{1}(r, s)=H_{2}(r, s)=(r-s)^{2}$ and $\rho(x)=|x|$ in Theorem 2.1. For any $T \geq 1$, let $n$ large enough such that $3 n \geq T$. Let $a=3 n, \ell=3 n+1$ and $b=3 n+2$, we can easily evaluate integral in (2.8) as

$$
\pi\left(9 n^{2}-6 n-\frac{44}{15}\right)>\frac{1}{15} \pi>0, \quad n>1 .
$$

Thus, in view of Theorem 2.1, we may conclude that Eq.(2.36) is oscillatory. In fact, if $y(x)$ is a solution of Eq.(2.36), then $y(x)$ has at least one zero in each annulus $\Omega(3 n, 3 n+2)$ for any integer $n \geq 1$.

Example 2.2. Consider the 2-dimensional Lapalacin equation

$$
\triangle y+b_{1}(x) \frac{\partial y}{\partial x_{1}}+b_{2}(x) \frac{\partial y}{\partial x_{2}}+\frac{\delta}{|x|^{2} \ln ^{2}|x|}\left(y+y^{3}\right)=0,
$$

where $x \in \Omega(1), \delta>1$ and $b_{1}, b_{2} \in \mathbf{C}^{\nu}(\Omega(1), \mathbb{R})$ may be not differentiable such that $|B(x)|^{2} \leq \delta /\left(|x|^{2} \ln ^{2}|x|\right)$. For Corollary 2.6, let $\rho(x)=1, \varepsilon=1 / 2$. A simple computation yields that

Note that

$$
\lambda(r)=2 \pi r, \quad \Lambda(r)=\frac{1}{2 \pi} \ln r, \quad \breve{c}(x) \geq \frac{\delta}{2|x|^{2} \ln ^{2}|x|} .
$$

$$
\int_{S(r)} \rho(x) \breve{c}(x) d \sigma \geq \frac{\pi \delta}{r \ln ^{2} r} \quad \text { and } \quad \frac{1}{\Lambda^{2}(r) \lambda(r)}=\frac{2 \pi}{r \ln ^{2} r} .
$$

For any $\delta>1$, there exists $u>1 / 2$ such that $\delta>2 u>1$. This means that (2.35) holds. By Corollary 2.6, Eq.(2.37) is oscillatory.

\section{References}

[1] D. Gilbarg and N. S. Trudinger, Elliptic Partial Differential Equations of Second Order, Spinger-Verlag, New York, 1983.

[2] G. H. Hardy, J. E. Littlewood, and G. Pólya, Inequalities, Second ed. Cambridge Univ. Press, Cambridge, 1988.

[3] Q. Kong, Interval criteria for oscillation of second-order linear ordinary differential equations, J. Math. Anal. Appl. 229 (1999), no. 1, 258-270.

[4] - Oscillation of second order matrix differential systems, Differential Equations Dynam. Systems 8 (2000), no. 2, 99-110.

[5] R. Mařík, Riccati-type inequality and oscillation criteria for a half-linear PDE with damping, Electron. J. Differential Equations 2004 (2004), no. 11, 17 pp.

[6] _ Ordinary differential equations in the oscillation theory of partial half-linear differential equation, J. Math. Anal. Appl. 338 (2008), no. 1, 194-208. 
[7] E. S. Noussair and C. A. Swanson, Oscillation of semilinear elliptic inequalities by Riccati transformations, Canad. J. Math. 32 (1980), no. 4, 908-923.

[8] Ch. G. Philos, Oscillation theorems for linear differential equations of second order, Arch. Math. (Basel) 53 (1989), no. 5, 482-492.

[9] C. A. Swanson, Semilinear second-order elliptic oscillation, Canad. Math. Bull. 22 (1979), no. 2, 139-157.

[10] A. Tiryaki and A. Zafer, Interval oscillation of a general class of second-order nonlinear differential equations with nonlinear damping, Nonlinear Anal. 60 (2005), no. 1, 49-63.

[11] Z. Xu, Oscillation of second order nonlinear elliptic differential equations, Kyungpook Math. J. 46 (2006), no. 1, 65-77.

[12] _ Oscillation criteria for second order elliptic equations with damping term, Util. Math. 74 (2007), 131-144.

[13] - Fite and Kamenev type oscillation criteria for second order elliptic equations, Ann. Polon. Math. 92 (2007), no. 3, 199-214.

[14] - Oscillation theorems for damped elliptic differential equations of second order, Hiroshima Math. J. 38 (2008), no. 1, 1-17.

[15] Z. Xu, B. Jia, and D. Ma, Oscillation theorems for elliptic equations with damping, Appl. Math. Comput. 156 (2004), no. 1, 93-106.

[16] Z. Xu and H. Xing, Domain criteria for oscillation of second-order damped elliptic equations, Acta Math. Sci. Ser. A Chin. Ed. 25 (2005), no. 3, 374-380.

[17] Z. Zheng, "Note on Wong's paper", J. Math. Anal. Appl. 274 (2002), no. 1, 466-473.

[18] R.-K. Zhuang and Z.-A. Yao, Some new oscillation criteria for second order elliptic equations with damping, Ann. Polon. Math. 86 (2005), no. 1, 31-42.

School of Mathematical Sciences

South China Normal University

Guangzhou, 510631, P. R. China

E-mail address: xuzhit@126.com 